Optical Society of America. Bing Yu, Dae Woong Kim, Jiangdong Deng, Hai Xiao, and Anbo Wang, "Fiber Fabry-Perot Sensors for Detection of Partial Discharges in Power Transformers," Appl. Opt. 42, 3241-3250 (2003). doi: 10.1364/ao.42.003241

\title{
Fiber Fabry-Perot sensors for detection of partial discharges in power transformers
}

\author{
Bing Yu, Dae Woong Kim, Jiangdong Deng, Hai Xiao, and Anbo Wang
}

\begin{abstract}
A diaphragm-based interferometric fiber optic sensor that uses a low-coherence light source was designed and tested for on-line detection of the acoustic waves generated by partial discharges inside high-voltage power transformers. The sensor uses a fused-silica diaphragm and a single-mode optical fiber encapsulated in a fused-silica glass tube to form an extrinsic Fabry-Perot interferometer, which is interrogated by low-coherence light. Test results indicate that these fiber optic acoustic sensors are capable of faithfully detecting acoustic signals propagating inside transformer oil with high sensitivity and wide bandwidth. (C) 2003 Optical Society of America
\end{abstract}

OCIS codes: $\quad 060.2370,120.2230,120.1880$.

\section{Introduction}

Power transformers are the most critical and expensive components in the power industry. The occurrence of partial discharges (PDs) within transformers may lead to insulation breakdown and catastrophic failures whose effects may be large in both safety and financial terms. The cost of each failure can easily drive the total cost of a single transformer failure to more than $\$ 10$ million. Hence it is important that PD activity be monitored and studied to detect incipient insulation problems, to prevent catastrophic failures and to avoid extensive costs.

The use of electrical, chemical, and acoustic measurements to detect PDs inside power transformers has been extensively studied. The electrical method may provide accurate recordings of PDs in laboratory conditions but is difficult to apply in the field for in-service transformers because of the high level of electromagnetic interference (EMI) and the impossibility of achieving accurate calibrations. Current chemical methods detect PDs in a transformer by taking gas or oil samples from the transformer. More-recent research includes the development of

When this research was performed, the authors were with the Center for Photonics Technology, Virginia Polytechnic Institute and State University, Blacksburg, Virginia 24061-0287. J. Deng is now with Nanoopto Corporation, Somerset, N.J. 08873. H. Xiao is now with Agere Systems, Inc., Allentown, Pennsylvania 18109. B. Yu's e-mail address is biyu@vt.edu.

Received 16 September 2002; revised manuscript received 21 January 2003.

0003-6935/03/163241-10\$15.00/0

(C) 2003 Optical Society of America gas sensors and their application for on-line gas monitoring. ${ }^{1-3}$ One major problem associated with chemical methods is that there is a long delay between the initiation of a PD source and the accumulation of enough gas to be detectable. For electrical and chemical methods a further limitation is that it is generally not possible to determine the exact location of a detected PD source, information that is also important in $\mathrm{PD}$ study. In general, a PD results in a localized, nearly instantaneous release of energy. It produces ultrasonic waves that propagate through the insulation oil of a transformer. An acoustic sensor can detect ultrasonic waves and generate useful information relevant to the PD sources. ${ }^{4-6}$ One obvious advantage of the acoustic methods is that by using them one can locate the position of a PD by studying the phase delay or the amplitude attenuation of the acoustic waves. Furthermore, acoustic methods have the potential advantage of better noise immunity in on-line PD detection applications.

One can achieve acoustic PD detection by mounting piezoelectric acoustic sensors externally on the walls of a power transformer, and often a suitable coupler is used to enhance the acoustic wave's coupling to the sensors. An externally mounted piezoelectric acoustic sensor offers the advantages of easy installation and replacement. However, a piezoelectric sensor may suffer from degeneration of the signal-to-noise ratio caused by environmental noises such as EMI and corona effects. Another possible disadvantage associated with an externally mounted piezoelectric sensor is that the multiple paths of acoustic wave transmission make locating the exact positions of partial discharges difficult. ${ }^{7,8}$ It is thus desirable to 
have sensors that can function reliably inside a transformer, even deep within the transformer windings, to pick up clean PD-induced acoustic signals. For the sake of safety and easy installation these sensors have to be chemically inert, electrically nonconducting, passive, and small in size.

Optical fiber-based sensors have been shown to be attractive devices with which to measure a wide range of physical and chemical parameters because the sensors have a number of inherent advantages, including small size, light weight, high sensitivity, high frequency response, electrical nonconductivity, and immunity to EMI noise. ${ }^{9}$ These advantages make optical fiber sensors excellent candidates for PD detection. Fiber optical acoustic sensors have been shown useful in many applications, such as underwater hydrophones, ${ }^{10,11}$ material property analysis, civil structure nondestructive diagnosis, ${ }^{12,13}$ vehicle detection and traffic monitoring, ${ }^{14}$ and partial-discharge detection. ${ }^{15,16}$ Early fiber optic sensors for acoustic signal detection were based mostly on fiber optic intrinsic interferometers such as all-fiber Michelson interferometers and MachZehnder interferometers.

These intrinsic fiber sensors usually use singlemode fiber and coherent sources, such as lasers. The light from a source is split into two fibers with equal intensity by a 3-dB fiber coupler. One fiber, referred to as the sensing arm, is exposed to the acoustic signal, and the other, referred to as the reference arm, is shielded from the impact of the acoustic wave. Either the reflections (Michelson) or the transmissions (Mach-Zehnder) of the light beams propagating in the two arms are recombined to generate interference signals that are modulated by the acoustic waves. The intrinsic fiber interferometric sensors have shown high sensitivity when a long fiber was used in the sensing arm. However, they suffer from the fringe fading problems that result from random polarization rotation. They are also unstable because of drift in the source wavelength and temperature-induced path-length changes.

More recently, fiber optic extrinsic Fabry-Perot interferometric (EFPI) sensors have been under development for acoustic-signal detection. ${ }^{17-20}$ Fiber EFPI sensors are fabricated with a small sensing element known as a Fabry-Perot cavity formed by two parallel reflecting surfaces. Compared to Michelson and Mach-Zehnder fiber sensors, EFPI sensors are compact in size and therefore achieve virtually single-point measurement. More importantly, those random polarization rotation and phase changes that are environmentally induced in the fiber connecting the optical source, the sensor head, and the detectors are common mode and therefore do not affect the signal phases.

The Center for Photonics Technology of the Virginia Polytechnic Institute and State University (CPT VT) has a research group experienced in the use of fiber optic EFPI sensors in harsh environments and has demonstrated a diaphragm-based EFPI prototype sensor system that uses a distributed- feedback laser source for on-line detection of partial discharges in power transformers. ${ }^{21}$ The PD sensors are based on a Fabry-Perot (F-P) interferometer that comprises a diaphragm and the end face of a single-mode fiber. The vibration of the diaphragm caused by PD-generated acoustic waves operates the interferometer in the linear range of one of its interference fringes. EFPI sensors operating in the linear range eliminate the common problems, such as nonlinear transfer functions, complex signal processing, and directional ambiguity, of sensors' measuring changes in air gap change by fringe counting. However, the PD detection system does suffer from source power fluctuation caused by backreflection, initial quadrature-point ( $Q$-point) offset because of fabrication tolerance, operating-point drift caused by static pressure in the transformer oil, and temperature drift. Although demodulation by multiwavelength interrogation ${ }^{14,22}$ or spectral interrogation ${ }^{22}$ is successful in solving the nonlinear transfer function, directional ambiguity, and signal fading, neither approach is suitable for PD detection because it is possible that all channels of the multiwavelength approach are not at the $Q$ point and that neither method may have enough frequency response for PD detection.

Low-coherence light sources, such as LEDs and surface LEDs (SLEDs), have found increasing applications in fiber optic interferometric systems because of their advantages over laser sources in terms of increased unambiguous measurement range, insensitivity to environmental perturbations, high resolution, and large dynamic range. ${ }^{23}$ The treatment of a broadband source basically goes back to principles related by Born and Wolf. ${ }^{24}$ By use of splitspectrum demodulation, ${ }^{14,25,26}$ EFPI sensors with broadband sources have been used for the measurement of pressure, temperature, vibration, and acoustic waves. However, even this approach does not help much to maintain the $Q$ point in the center of the linear region of a diaphragm-based F-P sensor system.

A promising technique for controlling the $Q$ point is use of a tunable-bandpass filter for dynamic control of the operating point. This approach takes full advantage of the broadband source and may also compensate for the slow $Q$-point drift caused by initial offset, static oil pressure, or temperature effects. Because it is difficult to find any commercial tunable bandpass filters at $1300 \mathrm{~nm}$, the CPT-VT is developing two techniques for achieving tunable bandpass filters. Although some positive initial results have been obtained with both techniques, it will still be some time before they can be used in our PD detection system. In the meantime it is beneficial for us to investigate the feasibility of the developing techniques for $Q$-point control by studying PD detection with a broadband source and a nontunable bandpass filter. In this paper we describe and analyze the performance of a PD detection system with a lowcoherence source and a bandpass filter. 


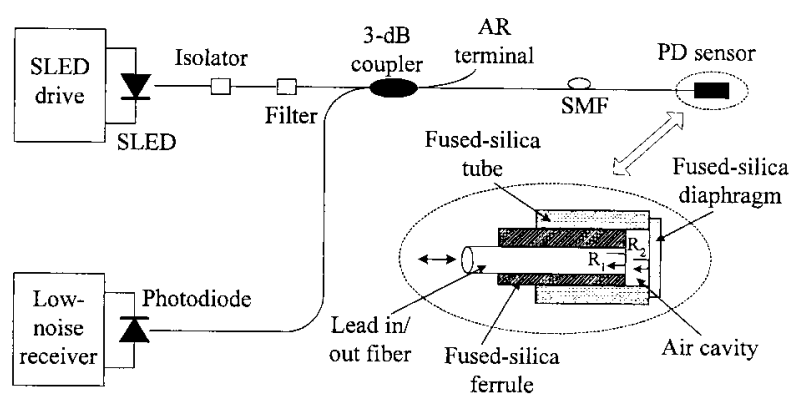

Fig. 1. Schematic of a fiber Fabry-Perot acoustic sensor system: SMF, single-mode fiber; AR, antireflection.

\section{Method of Operation}

The fiber optic acoustic sensor is illustrated schematically in Fig. 1. The system consists of a sensor probe, a SLED, a bandpass filter, a low-noise wideband optical receiver, and single-mode fibers linking the sensor probe and the optical receiver. The light from a 1300-nm high-power superluminescent LED is launched into an isolator and propagates along the fiber to the sensor head through a filter and a $2 \times 2$ 3 -dB coupler. A 1310-nm optical isolator was inserted just after the SLED to reduce optical feedback to the source, and an optic bandpass filter confined the spectrum within a certain range. As shown in the enlarged view of the sensor head (Fig. 1, inset), the lead in-out fiber and the thin silica glass diaphragm are bonded together in a cylindrical fusedsilica tube to form a F-P sensing element. The use of fused silica for all parts ensures that the temperature effects will be minimum. The incident light is first partially reflected $\left(R_{1}\right)$ at the end face of the lead in-out fiber. The remainder of the light propagates across the air gap to the inner surface of the diaphragm, where it is once again partially reflected $\left(R_{2}\right)$. The diaphragm etalon formed by the two surfaces of the diaphragm had to be carefully treated in the previous PD sensor system described earlier in this paper. Three reasons make it negligible in the new system that uses a SLED source and a bandpass: (1) The coherence length of the light is much shorter than that of the distributed-feedback laser, (2) coupling loss causes low fringe visibility and intensity; and (3) the refractive index of the environment is close to that of the diaphragm ( $\sim 1.48$ for transformer oil and $\sim 1.33$ for water). The multiple reflections travel back along the same lead-in fiber and through the same fiber coupler to the optical receiver.

The spectral distribution of a SLED may be described by a Gaussian function ${ }^{23}$ :

$$
f(\lambda)=\frac{1}{(2 \pi)^{1 / 2} \Delta \lambda} \exp \left[-\left(\lambda-\lambda_{0}\right)^{2} /\left(2 \Delta \lambda^{2}\right)\right]
$$

where $f(\lambda)$ is the spectral density, $\lambda_{0}$ is the central wavelength, and $\Delta \lambda=\Delta \lambda_{\text {FWHM }} /(8 \ln 2)^{1 / 2}$. Assuming that the fiber end and the diaphragm have the same reflectance $\left(R_{1}=R_{2}=R\right)$, the optical intensity $I_{r}(\lambda)$ received by the receiver at wavelength $\lambda$ is

$$
I_{r}(\lambda)=\left[\alpha I_{0} f(\lambda)\right] \frac{R^{2}\left[(1+\eta)-2 \eta^{1 / 2} \cos \left(4 \pi n_{0} L / \lambda\right)\right]}{1+\eta R^{2}-2 \eta^{1 / 2} R \cos \left(4 \pi n_{0} L / \lambda\right)},
$$

where $I_{0}$ is the SLED output power after the pigtail, $\alpha$ is the loss coefficient of the optical path (from the source to the sensor and from the sensor to the receiver, including the fiber, the isolator, the filter, and the couplers), $L$ is the air-gap length, $n_{0}=1$ is the refractive index of the air in the cavity, and $\eta$ is the coupling efficiency for the round trip between the fiber end and the diaphragm. Provided that the diaphragm is parallel to the fiber end, $\eta$ can be calculated by ${ }^{27}$

$$
\eta=1 /\left\{1+\left[2 \lambda_{0} L /\left(2 \pi n_{0} w^{2}\right)\right]^{2}\right\},
$$

where $w$ is the mode spot size of the single-mode fiber. Therefore the total optical power received by the receiver can be expressed as

$$
\begin{aligned}
I= & \int_{\lambda_{0}-\mathrm{BW} / 2}^{\lambda_{0}+\mathrm{BW} / 2} I_{r}(\lambda) \mathrm{d} \lambda \\
= & \frac{\alpha I_{0}}{(2 \pi)^{1 / 2} \Delta \lambda} \int_{\lambda_{0}-\mathrm{BW} / 2}^{\lambda_{0}+\mathrm{BW} / 2} \\
& \times \frac{R^{2}\left[(1+\eta)-2 \eta^{1 / 2} \cos \left(4 \pi n_{0} L / \lambda\right)\right]}{1+\eta R^{2}-2 \eta^{1 / 2} R \cos \left(4 \pi n_{0} L / \lambda\right)} \\
& \times \exp \left[-\left(\lambda-\lambda_{0}\right)^{2} /\left(2 \Delta \lambda^{2}\right)\right] \mathrm{d} \lambda,
\end{aligned}
$$

where BW is the total spectrum width of the interrogating light wave. The SLED used is SLED1300D20A from Opto Speed, which has a center wavelength of $1300 \mathrm{~nm}$ and $\Delta \lambda_{\text {FWHM }}$ of $35 \mathrm{~nm}$. Its spectrum spreads from 1200 to $1400 \mathrm{~nm}$, as shown in Fig. 2.

From Eq. (4), the interference of the multiple reflections produces almost sinusoidal intensity variations for a low-finesse F-P interferometer, defined as interference fringes, as the air gap is continually changed by acoustic wave pressure. Figure 3 gives the theoretical results from Eq. (4) normalized to the maximal optical intensity reflected from the F-P cavity, showing the change in air cavity length for a system without [Fig. 3(a)] and with [Fig. 3(b)] a bandpass filter, where the reflective surfaces are not coated $(R=3.5 \%)$. Obviously, the sensor with the natural spectrum of the SLED has a short coherent length and low visibility ( $\sim 25 \%$ for an air-gap length of $15 \mu \mathrm{m}$ ). These limitations require that the air cavity be shorter than $10 \mu \mathrm{m}$ to produce higher fringe visibility, which places more difficult manufacturing constraints on the sensor head. In contrast, the interference fringes have very good visibility $(\sim 65 \%$ at a cavity length of $15 \mu \mathrm{m}$ ) if $\Delta \lambda_{\text {FWHM }}$ is reduced to 20 $\mathrm{nm}$ by use of coarse wavelength-division multiplexing a bandpass filter. This use of a badnpass filter also 


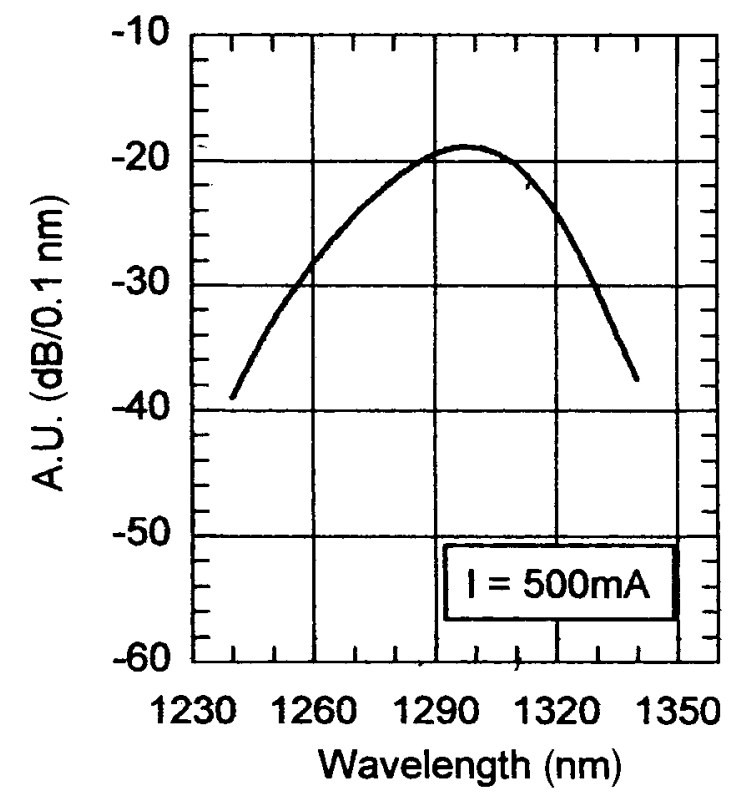

Fig. 2. Spontaneous-emission spectrum of SLED1300D20A.

significantly increases the fabrication tolerance at the expense of out-of-band optical power ( $~ 50 \%$ for the SLED1300D20A), which is acceptable in our system's power budget.

From Fig. 3, one period of fringe variation corresponds to an air-gap change of one half of the optical wavelength, which in our case is $\sim 0.65 \mu \mathrm{m}$. In principle, continuous tracking of phase changes in the interference fringes can yield information about airgap changes in the sensor element. The acoustic signal generated by partial discharges causes deflection of the diaphragm and modulates the sealed airgap length. The sensor therefore yields outputs that correspond to the applied acoustic signals. As in regular interferometers, the measurement will have ultrahigh sensitivity. However, the measurement will suffer from the disadvantages of sensitivity re-

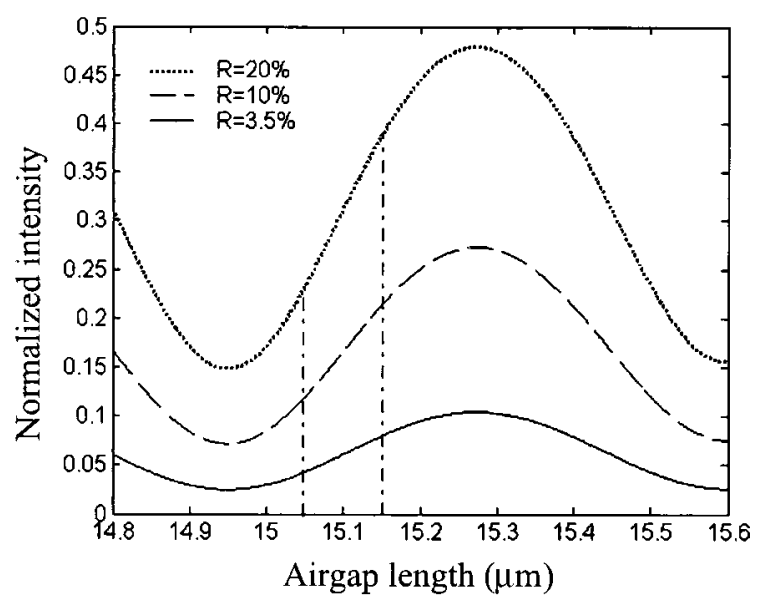

Fig. 4. Illustration of the linear operating range of the sensor's response curve; $R$ is the reflectivity.

duction and ambiguity in fringe direction when the sensor reaches peaks or valleys of the fringes. Sensitivity is reduced at peaks or valleys of the fringes because at those points the change in optical intensity is nearly zero for a small air-gap change. Ambiguity in fringe direction is defined as difficulty in determining whether the air gap is increasing or decreasing by detecting the optical intensity only. If a measurement starts with an air gap corresponding to the peak of a fringe, the optical intensity will decrease, regardless of whether the gap increases or decreases.

One approach to solving these problems is to design the sensor head such that the maximum air-gap change does not exceed the linear region of a halffringe. Figure 4 shows the interference fringes of sensor heads normalized to the optical intensity in the F-P cavity at reflectances $R=3.5 \%, 10 \%, 20 \%$ for changes in air-gap length from 14.8 to $15.6 \mu \mathrm{m}$. Choosing as the operating point $(Q$ point or initial air-gap length) the central point $\left(L_{0}\right)$ between a peak

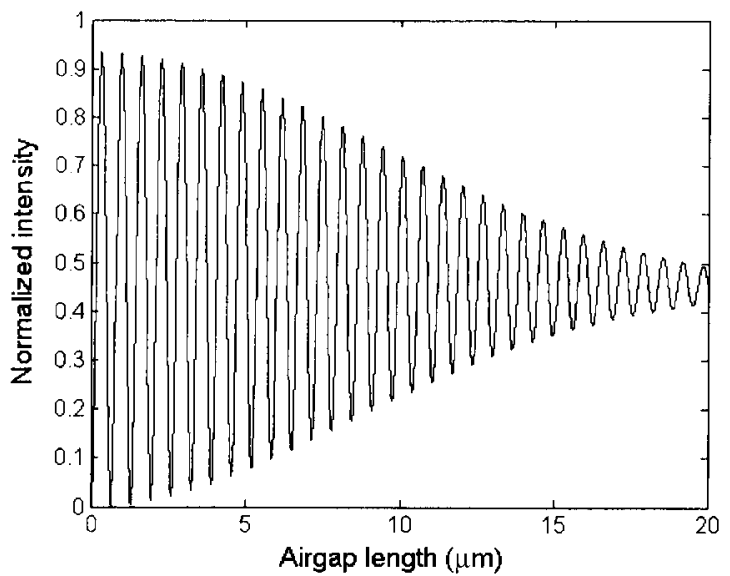

(a)

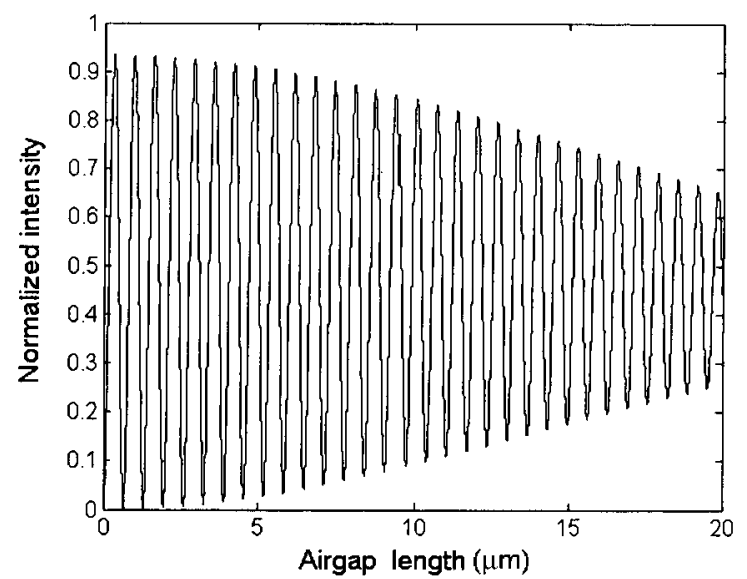

(b)

Fig. 3. Interference fringes of a low-finesse sensor (reflectivity $R=3.5 \%$ ) with a SLED source: (a) no filter, $\Delta \lambda_{\text {FwHM }}=35 \mathrm{~nm}$; (b) with a bandpass filter, $\Delta \lambda_{\mathrm{FWHM}}=20 \mathrm{~nm}$. 
Table 1. Fitting Parameters for Linear Regions with a Norm of Residuals of $10^{-3}$

\begin{tabular}{clccc}
\hline $\begin{array}{c}\text { Reflectivity, } \\
R(\%)\end{array}$ & \multicolumn{1}{c}{$S_{0}$} & \multicolumn{1}{c}{$C$} & $\begin{array}{c}Q \text { Point } \\
L_{0}(\mu \mathrm{m})\end{array}$ & $\begin{array}{c}\text { Linear Range, } \\
2 \Delta L(\mathrm{~nm})\end{array}$ \\
\hline 3.5 & 0.3777 & -5.6425 & 15.108 & 96 \\
10 & 0.96535 & -14.411 & 15.100 & 72 \\
20 & 1.6124 & -24.036 & 15.092 & 62 \\
\hline
\end{tabular}

and its neighbor valley for each sensor head, we may treat a region defined by $\left(L_{0} \pm \Delta L\right)$ (between the dotted-dashed vertical lines in Fig. 4) as a linear region, which can be fitted with Matlab by the following linear equation:

$$
I(L)=S_{0} L+C,
$$

where $I(L)$ is the optical intensity at air gap $L$ and $S_{0}$ and $C$ are constants. Obviously $S_{0}$ represents the fringe sensitivity of a sensor to a change in the air gap near its $Q$ point. For a norm of residuals of $10^{-3}$, the fitting parameters were calculated and are listed in Table 1 . The increase in reflectivity $R$ from $3.5 \%$ to $20 \%$ results in an increase in sensitivity of a factor of 4.27 , but the decrease in linear operation ranges from 96 to $62 \mathrm{~nm}$. In addition, the $Q$ point of the linear region drifts toward the valley of the fringes when the reflectance is increased. However, visibility and absolute amplitude increase with increased reflectivity.

In this linear region the ac electrical output of the sensor $V(P)$, is proportional to the air-gap change $y_{0}(P)$ that is caused by acoustic pressure $P$ and can be expressed as

$$
V(P)=G \Re \alpha S_{0} I_{\text {ac }}\left[L_{0}+y_{0}(P)\right]=S_{\text {oe }} y_{0}(P),
$$

where $S_{0}$ is the fringe sensitivity, i.e., the slope of the fringes in the linear regions; $\Re$ is the responsivity of the InGaAs photodetector, $\sim 0.9$ at $1300 \mathrm{~nm} ; G$ is the total gain of the optical receiver; $I_{\mathrm{ac}}\left[L_{0}+y_{0}(P)\right]$ is the ac component of the received optical signal; $y_{0}(P)$ is the diaphragm deflection caused by acoustic pressure P; $S_{\text {oe }}$ is the total optical and electrical sensitivity with respect to the air-gap change; and

$$
S_{\text {oе }}=G \Re \alpha S_{0} .
$$

\section{Diaphragm-Design Considerations}

Diaphragms and membranes have found extensive applications in pressure and acoustic wave measurements in the mechanical and microelectromechanical system sensor industries. As shown in the inset of Fig. 1, we fabricated a sensor head by thermally bonding a fiber, a ferrule, a silica tube, and a silica diaphragm together to form a sealed F-P interferometer. The diaphragm vibrates in the presence of an acoustic wave, which imposes a dynamic pressure on it. The diaphragm's design is probably the most critical part of the sensor for efficient acoustic wave detection. Sensitivity, linearity, frequency response, temperature dependence, and size of the sensor head are directly related to the properties of the

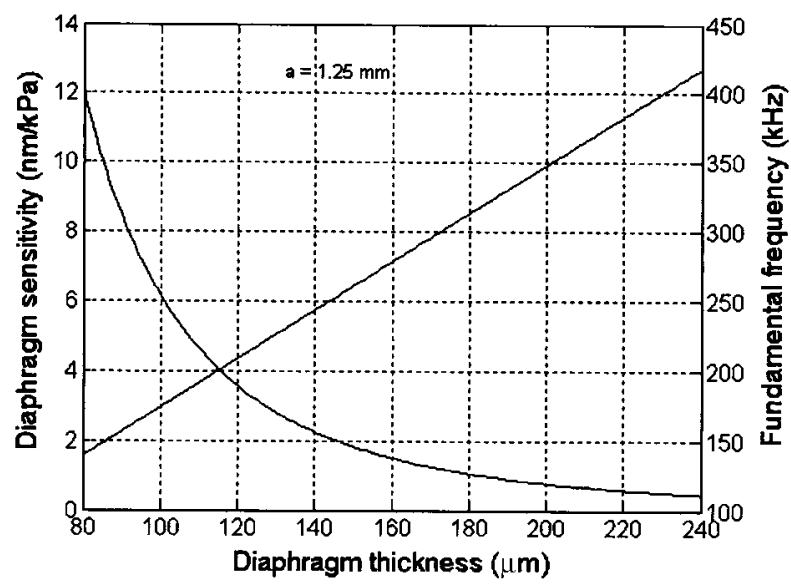

Fig. 5. Sensor's sensitivity and natural frequency versus diaphragm thickness for $a=1.25 \mathrm{~mm}$.

diaphragm. For the sake of extremely low dependence on temperature, we selected fused silica, the same material as used in the single-mode optical fiber, as the material to be used for the ferrule, the tube, and the diaphragm, as mentioned above. However, the shape and the size of the diaphragm are yet to be determined by the sensitivity and bandwidth requirements of the system.

\section{A. Sensitivity}

The diaphragm will be deflected whenever there is a differential pressure $P$ between the inside and the outside of the sealed cavity. The out-of-plane deflection of the diaphragm, $y$, is a function of the pressure difference at any radial position, $r$. With the assumption of uniform diaphragm thickness, small deflection, infinitely rigid clamping about the periphery of the diaphragm, and perfectly elastic behavior, which is almost true for the fused-silica diaphragm and our bonding method, the deflection under pressure difference $P$ is can be expressed as ${ }^{28}$

$$
y(P)=\frac{3\left(1-\mu^{2}\right)}{16 E h^{3}}\left(a^{2}-r^{2}\right)^{2} P
$$

where $\mu$ is Poisson's ratio ( $\mu=0.16$ for fused-silica glass 7940 at $25^{\circ} \mathrm{C}$ ), $E$ is Young's modulus of the silica glass material $\left(E=7.24 \times 10^{10} \mathrm{~Pa}\right.$ or $73.5 \times 10^{8}$ $\mathrm{kg} / \mathrm{m}^{2}$ at $25^{\circ} \mathrm{C}$ ), $a$ is the radius of the diaphragm defined by the inner diameter of the glass tube, and $h$ is the thickness of the diaphragm. In our sensor configuration the fiber is positioned at the central part of the diaphragm such that only the center deflection $y_{0}$ is of interest. The sensitivity of the diaphragm of a fused-silica diaphragm is given by

$$
\delta=\frac{y_{0}(P)}{P}=2.524 \times 10^{-6} \frac{a^{4}}{h^{3}}[\mathrm{~nm} / \mathrm{kPa}],
$$

where $y_{0}$ is given in nanometers, $a$ and $h$, in micrometers; and $P$, in kilopascals. Figure 5 shows a typical 
sensor sensitivity curve at $a=1.25 \mathrm{~mm}$. Substituting Eq. (9) into Eq. (6) yields

$$
V(P)=S_{T} P,
$$

where

$$
S_{T}=2.524 \times 10^{-9} \frac{a^{4}}{h^{3}} S_{\text {oe }}=2.524 \times 10^{-9} \frac{a^{4}}{h^{3}} G \Re \alpha S_{0}
$$

is the total sensitivity of the sensor system.

Obviously, $V(P)$ is proportional to the applied acoustic pressure generated by partial discharge when the sensor operates in the linear region of an interference fringe. This linear relationship makes the signal processing of our fiber optic PD sensors much simpler than those of other F-P sensors that use a fringe counting or a spectrum demodulation approach. From Eq. (11), the total sensitivity of the system is determined by the parameters of the diaphragm, the gain of the amplifier, the loss of the optical system, and the fringe sensitivity. The diaphragm's parameters are also related to the sensor's frequency response. Larger amplifier gain may increase the total sensitivity but is limited by the bandwidth requirement and signal-to-noise ratio. The use of a bandpass filter can increase the visibility of interference fringes and therefore increase the useful ac optical intensity. Another effective way to increase sensitivity is to use diaphragms with reflective coatings. As shown in Fig. 4, fringe sensitivity is determined by reflectance $R$. A second benefit of using a coated diaphragm is that coupling coefficient $\alpha$ can be increased at the same time. However, when the reflectance is increased, the finesse of the F-P interferometer is also increased. This results in steeper fringes and shorter linear regions on each fringe and thereby requires that $R$ be less than $20 \%$ unless better strategies are used to keep the sensor working in a smaller linear region.

\section{Frequency Response}

For partial-discharge detection we suggest that the sensitive frequency of the sensor system is in the range $20-200 \mathrm{kHz},{ }^{4-8}$ basically because noise induced in transformers by magnetostriction usually exists in the low-frequency range below $60 \mathrm{kHz}$, and the acoustic emission frequency of a PD is mostly $\sim 150 \mathrm{kHz}^{6}$

To respond faithfully to dynamic acoustic wave pressure requires that the resonant frequency of the diaphragm be higher than the highest applied frequency. For reference only, and subject to the same assumptions for the calculation of sensitivity, the undamped $n$-order resonant frequency of a rigidly clamped diaphragm can be expressed as ${ }^{29}$

$$
f_{n}=\frac{\alpha h}{2 \pi \alpha^{2}}\left[\frac{g E}{12\left(1-\mu^{2}\right)}\right]^{1 / 2},
$$

where $\alpha$ is a constant that is related to the vibrating modes of the diaphragm and takes a value of 10.21 for the fundamental mode $(n=0), g=9.815 \mathrm{~m} / \mathrm{s}^{2}$ is the gravitational constant, and $w$ is the weight (density) of the diaphragm material. For a fused-silica glass diaphragm at $25^{\circ} \mathrm{C}, w$ is $2.20 \times 10^{3} \mathrm{~kg} / \mathrm{m}^{3}$. The frequency response of the sensor can thus be obtained as

$$
f_{0}=2.72 \times \frac{h}{a^{2}}[\mathrm{kHz}],
$$

where $h$ is given in micrometers and $a$ in millimeters. As indicated by Eq. (13), the frequency response is proportional to the thickness of the diaphragm and is inversely proportional to the square of the effective diaphragm radius. The fundamental natural frequency of a fused-silica diaphragm with an effective diameter of $2.5 \mathrm{~mm}$ is given in Fig. 5 for various diaphragm thicknesses. In practice, Eq. (13) may not be accurate for a diaphragm-based PD sensor because of the presence of mineral oil. In a fluid, the natural frequency should be modified by a factor of $1 /(1+\beta)^{1 / 2}, 29$ where $\beta=0.669 w_{1} R / w h$ and $w_{1}=$ $0.85 \times 10^{3} \mathrm{~kg} / \mathrm{m}^{3}$ is the density of the transformer oil. For a $2.5-\mathrm{mm}$ diameter and a $150-\mu \mathrm{m}$ diaphragm, the modification factor is $\sim 0.56$. However, considering that only one side of the diaphragm is immersed in mineral oil, and in a condition in which a small volume of air is trapped behind the diaphragm and the volume change caused by deflection is not insignificant compared with the total volume of the trapped air, a slight increase in the resonant frequency is possible, as the trapped air might act as a stiffening spring, as indicated in Ref. 29, and the modification factor could be even closer to 1. Therefore Eq. (13) can still be used to estimate the fundamental natural frequency in the diaphragm design.

In partial-discharge detection, we suggest that the sensitive frequency of the sensor system is in the range $20-200 \mathrm{kHz}$. A trade-off must be made between high sensitivity and high frequency response of the sensor when one is selecting the geometric size of the diaphragm. For example, if a diaphragm of $a=$ $1.25 \mathrm{~mm}$ and $h=125 \mu \mathrm{m}$ is chosen, a fundamental frequency of $220 \mathrm{kHz}$ and a diaphragm sensitivity of $3.5 \mathrm{~nm} / \mathrm{kPa}$ may be obtained. A maximum acoustic pressure of $10.3 \mathrm{kPa}$ will result if a $10 \%$ reflective coating $(\Delta L= \pm 36 \mathrm{~nm})$ is deposited both on the diaphragm and on the fiber. An acoustic-pressure resolution of $10 \mathrm{~Pa}$ may be obtained if a system signal-to-noise ratio of $60 \mathrm{~dB}$ is achievable.

\section{Sensor Fabrication}

Several sensor heads have been fabricated either by fusion bonding with borosilicate powders or by epoxy bonding of a single-mode fiber, a silica glass ferrule, a silica glass tube, and a thin silica diaphragm, as shown in Fig. 1. Fusion bonding has the advantage of a low temperature coefficient but the disadvantage of difficult air-gap control and possible damage to the reflective coatings. Bonding with epoxy is simpler and more easily controllable but has a higher sensitivity to temperature. 


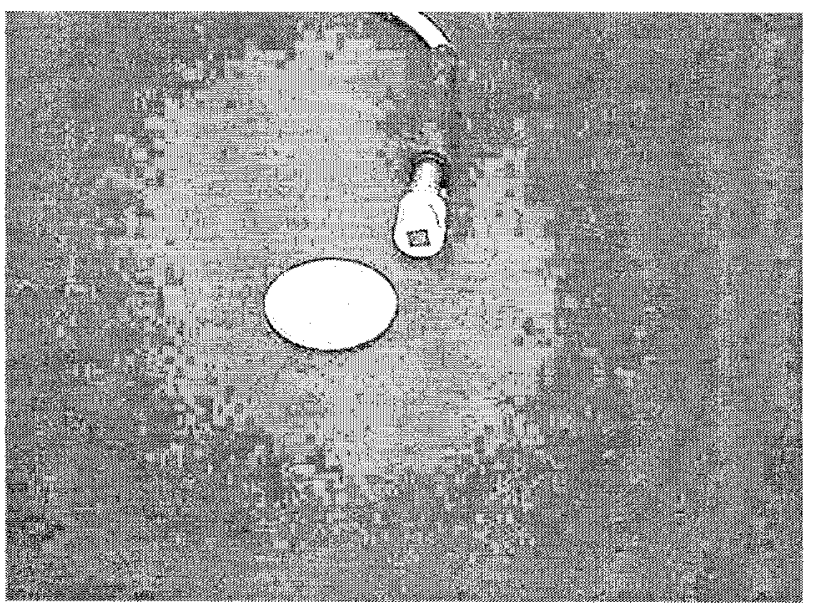

Fig. 6. Photograph of a fiber PD sensor.

The ferrules used have an inner diameter of 130 $\mu \mathrm{m}$ and an outer diameter of $2.50 \mathrm{~mm}$; the tubes have an inner diameter of $2.52 \mathrm{~mm}$ and an outer diameter of $6 \mathrm{~mm}$. The diaphragms have two different thicknesses, 125 and $250 \mu \mathrm{m}$, with $10 \%$ coatings. A sample of a finished sensor is shown in Fig. 6. In the sensor fabrication, a monitoring system consisting of a broadband light source, a $2 \times 2$ coupler, and an optical spectrum analyzer was been used to monitor the air-gap length. The initial air gap between the fiber and the inner surface of the silica diaphragm was adjusted to produce the highest interference fringe visibility. The initial operating point was also adjusted to the central point of a fringe for linear operation and the highest detection sensitivity. For those sensors to be used in transformer oil, the original air cavity length must be a bit larger or smaller than the optimal length to compensate for the static pressure caused by the oil. The small air-gap difference from the optimal point depends on the mounting depth of the sensor in the transformer oil.

However, no matter how well the initial air gap can be controlled, temperature drift and static oil pressure may drive the operating point away from the $Q$ point. Inasmuch as all parts of the sensors are made from fused-silica glass, the temperature coefficient of a fusion-bonded sensor can be as small as $0.01 \mathrm{~nm} /{ }^{\circ} \mathrm{C}$ for a cavity length of $20 \mu \mathrm{m}$ and therefore can be neglected. Unfortunately, the oil pressure can be as high as $58 \mathrm{kPa}(\sim 8.5 \mathrm{psi})$ and may drive the operating point to a peak or a valley of an interference fringe. A promising approach to dynamic control of the operating point in the linear region while high fringe visibility is maintained is to use a tunable bandpass optical filter. Initial laboratory experimental results with underdeveloping tunable optical bandpass filters have already proved that dynamic control of the $Q$ point is possible.

\section{Experimental Results and Discussion}

A prototype system of this design, shown in Fig. 7, was built and tested to demonstrate the feasibility of on-line detection of partial discharges in power trans-

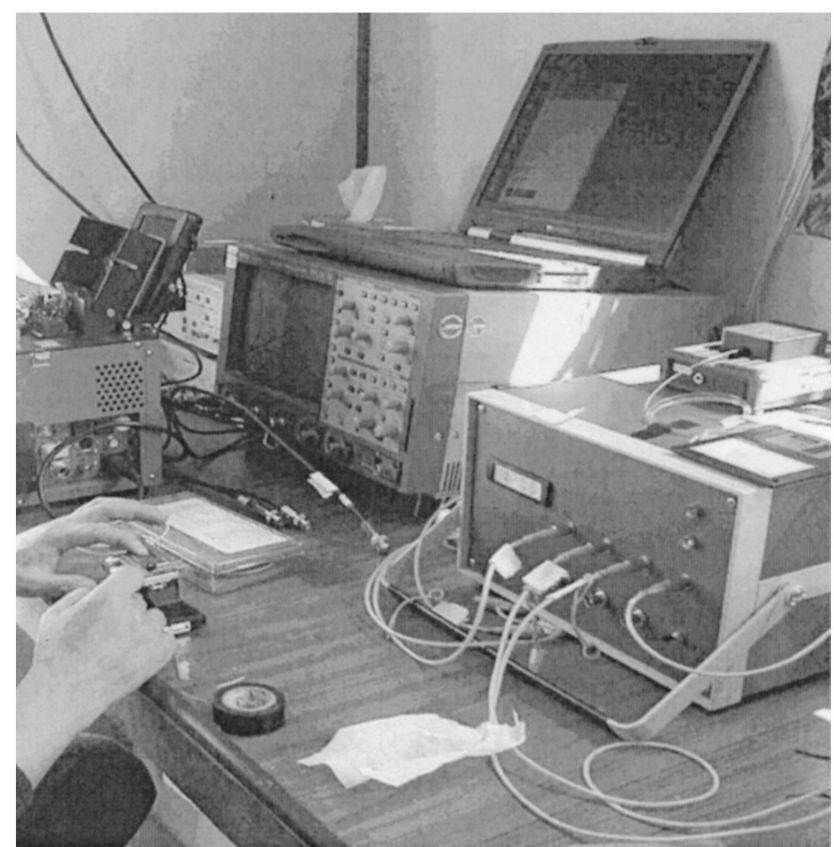

Fig. 7. Prototype of a three-channel fiber optic PD detection system.

formers. A $20-\mathrm{mW}$ SLED centered at $1300 \mathrm{~nm}$ with spectrum width $\Delta \lambda_{\text {FWHM }}=35 \mathrm{~nm}$ is used as the source. This high-power SLED supports three PD sensor channels in the prototype. Three high-speed InGaAs detectors are used to detect the optical signal from each sensor, and the output is fed into three high-gain wideband transimpedance amplifiers. The frequency response of the electronic circuits is limited within a range of $20-300 \mathrm{kHz}$, defined by additional bandpass filters.

We set up an experimental system to evaluate the performance of the diaphragm-based PD sensors for acoustic wave detection. As shown in Fig. 8, a fiber PD sensor was installed in water at one end of a polyvinyl chloride (PVC) tube facing a piezoelectric (PZT) transducer (Physical Acoustic Corporation) at the other end of the PVC tube. The transducer has an acoustic bandwidth of $100 \mathrm{kHz}-1 \mathrm{MHz}$. The perturbed angular correlation (Physical Acoustic Corporation) C-101_HV pulser has an output pulse rise time of $20 \mathrm{~ns}$ and a pulse width of $100-500 \mathrm{~ns}$. We

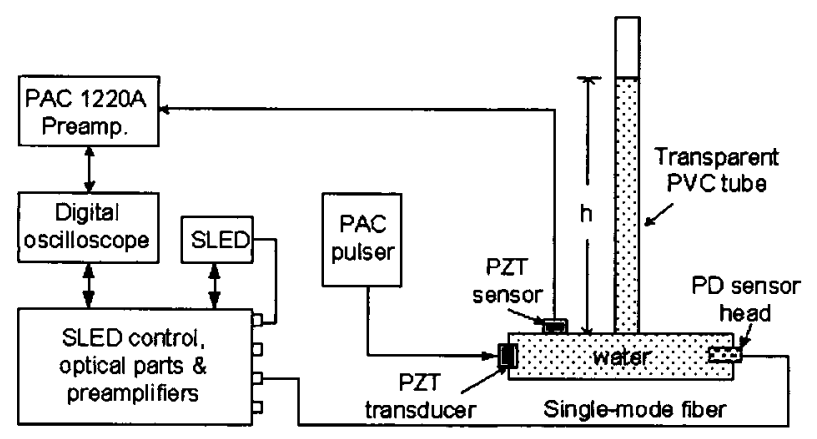

Fig. 8. Schematic of the test setup for PD sensor evaluation. 


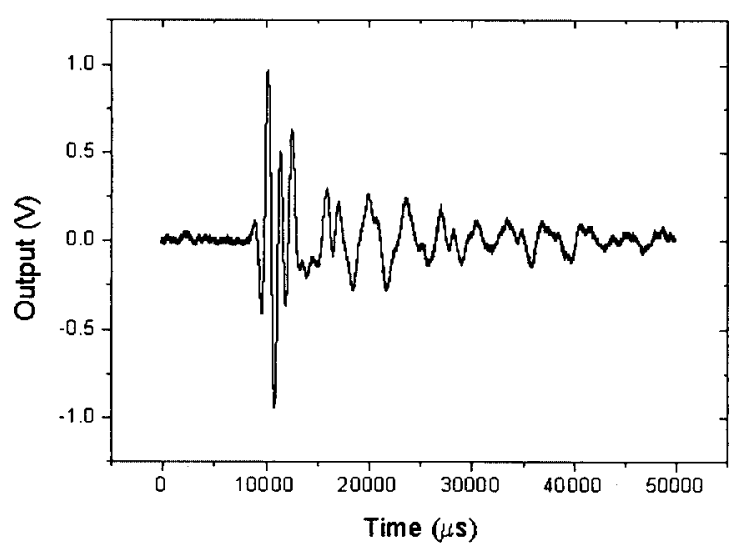

(a)

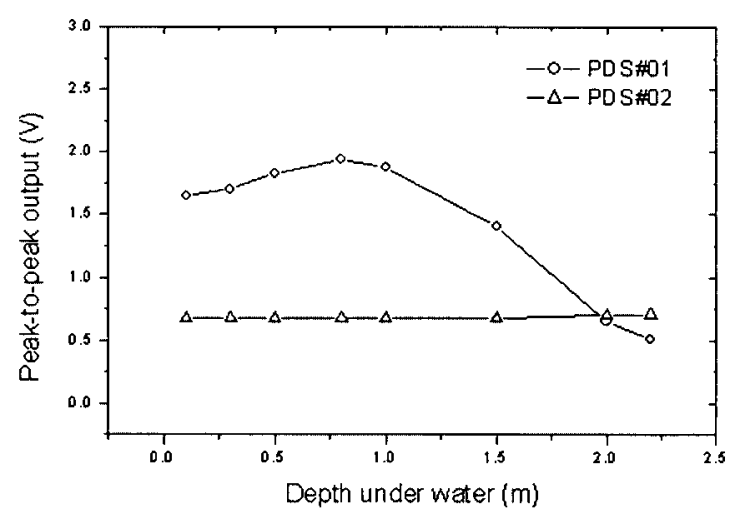

(c)

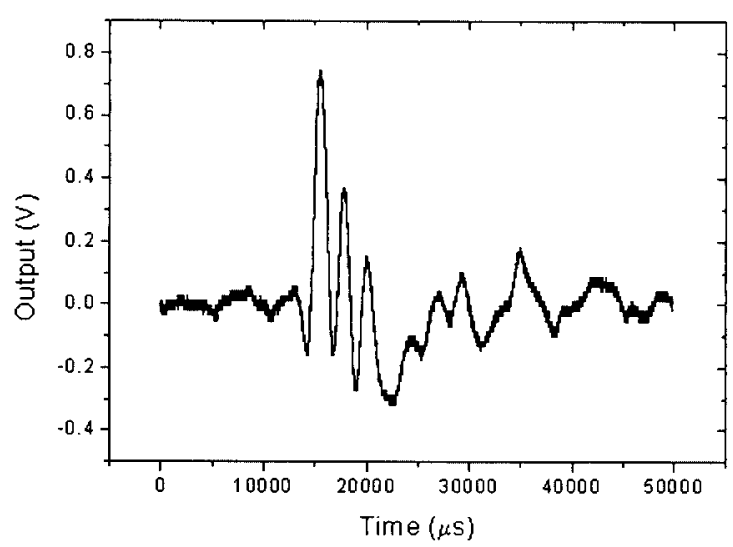

(b)

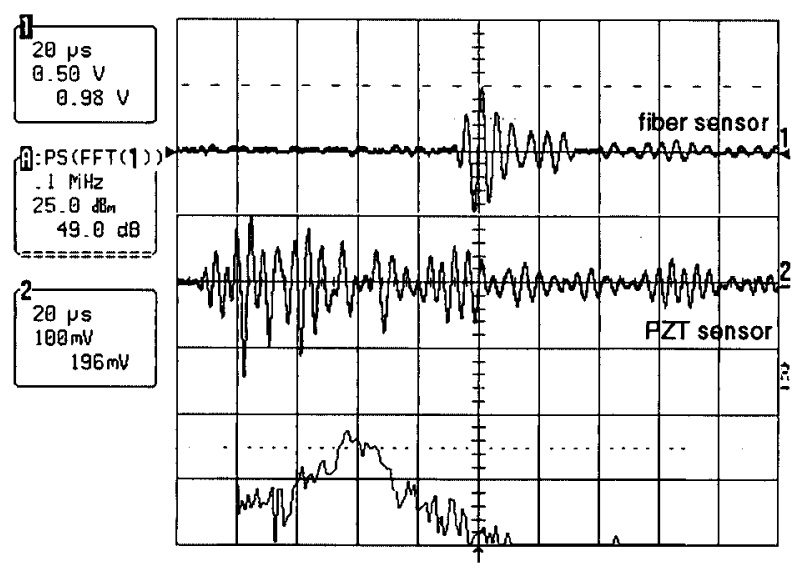

(d)

Fig. 9. Laboratory simulation of acoustic wave detection: (a) $h=1.0 \mathrm{~m}$, (b) $h=2.0 \mathrm{~m}$, (c) peak-to-peak output with change in static pressure, (d) comparison of a fiber sensor with a PZT sensor and the fast Fourier transform (FFT) of the output from the fiber sensor.

can adjust the static water pressure by varying the height of the water column from 0.1 to $2.2 \mathrm{~m}$, simulating the static pressure of a power transformer in the mineral oil. The distance from the PD sensor to the PZT is $\sim 18 \mathrm{~cm}$. As a reference, we attached another PZT was attached to the outside of the PVC tube as an acoustic sensor. Because the signal coupling is not efficient, the PZT was mounted closer to the source than the fiber sensor. This arrangement was chosen because it represents many practical PD test situations in which PZT sensors are attached to the sidewalls of a transformer whereas fiber sensors can be inserted into the transformer tank.

A LeCroy two-channel digital oscilloscope is used to display and store the acoustic signals captured by the sensors. The test results for the two fiber sensors, PDS\#1 with a $125-\mu \mathrm{m}$ diaphragm and PDS\#2 with a $250-\mu \mathrm{m}$ diaphragm, were recorded and are shown in Fig. 9. Figures 9(a) and 9(b) show the waveforms captured by PDS\#1 depths of 1.0 and $2.0 \mathrm{~m}$, respectively, and Fig. 9(c) shows the peak-to-peak amplitudes with the static water pressure change for sensors PDS\#1 and PDS\#2. Obviously, sensor PDS\#1 is more sensitive than sensor PDS\#2 to the acoustic waves and to changes in static pressure.
These results agree well with the sensitivity calculation given in Fig. 5. When sensor PDS\#1 operates near its optimal point $(h=0.8 \mathrm{~m})$, it has maximal sensitivity and the best linearity. The measured linear range for PDS\#1 is $\sim 1 \mathrm{~m}$, or $9.8 \mathrm{kPa}$, which is very close to the value of $10.3 \mathrm{kPa}$ calculated in Section 3. However, as the increasing static water pressure drives the operating point closer to a peak or a valley of an interference fringe, sensitivity is reduced and the nonlinearity effect becomes significant. This can clearly be seen from the shapes of the waveforms captured by sensor PDS\#1 at $h=1.0 \mathrm{~m}$ in Fig. 9(a) and at $h=2.0 \mathrm{~m}$ in Fig. 9(b). Comparing the amplitudes of the waveforms in Fig. 9(a) with the curve for PDS\#1 in Fig. 9(c) and noting that the amplifier gain in the latter figure is approximately a quarter of that for Fig. 9(a), we know that the peakto-peak acoustic pressure $(< \pm 1.0 \mathrm{~V})$ is only one half of the linear range $(\sim 1.0 \mathrm{~V} \times 4)$. This means that at $h=1.0 \mathrm{~m}$ sensor PDS\#1 faithfully responds to the dynamic acoustic waves generated by the PZT.

For Fig. 9(d) the signals were detected by sensor PDS\#1 and the PZT sensor for the same acoustic wave shock. The fast Fourier transform of the signal from PDS\#1 indicates that the central frequency 


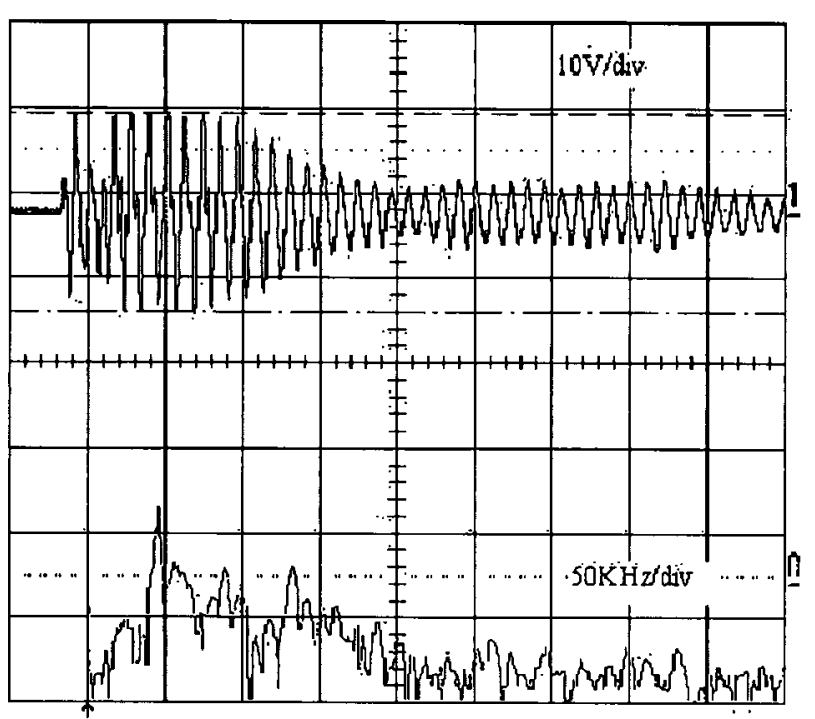

Fig. 10. Top, PD signal and bottom, its spectrum generated by a car starter.

is $\sim 190 \mathrm{kHz}$. A comparison of these two timedomain waveforms generates interesting information: First, the waveform from PDS\#1 appears to be similar to the leading end of the waveform from the PZT sensor. This means that both of them picked up the acoustic wave from the PZT source. Second, multiple reflections are coupled into the PZT sensor, cause degeneration of the received signals, and make it impossible to determine how long a PD will last, whereas the fiber sensor in the PVC tube picked up stronger and cleaner acoustic signals. Finally, there is a clear time delay of $80 \mu \mathrm{s}$ between the two wave fronts, which is caused by the $12-\mathrm{cm}$ distance between those two sources. The speed of an acoustic wave is $\sim 1500 \mathrm{~m} / \mathrm{s}$ in water.

To further investigate the feasibility of real PD detection with a diaphragm-based fiber sensor we used a car starter as a PD source in our lab. We used both a fiber sensor that we made and a PZT sensor from Physical Acoustic Corporation to measure the PD-generated acoustic waves. Two groups of experiments were performed. In the first group, both the car starter and the sensors were placed close to the signal-processing systems. Strong EMI noise was received by both systems, and the acoustic wave was immersed in the noise. In the second experiment the sensors were enclosed together with the starter in an aluminum box $20 \mathrm{~m}$ away from the signal-processing system. Considering that acoustic waves have much higher attenuation in air than in liquid, we mounted the sensors only $2 \mathrm{~cm}$ from the sparker. In this way the fiber sensor caught a strong acoustic wave with peaks at $45 \mathrm{kHz}$ and harmonic frequencies as shown in Fig. 10; the EMI noise was less than $100 \mathrm{mV}$. In the meantime, the PZT sensor still picked up strong EMI noise because of the electrical cable connection between the sensor and the signal-processing system. This comparison proves one of the advantages of fiber sensors compared with electronic sensors.

\section{Conclusion}

In summary, a diaphragm-based fiber optic FabryPerot sensor system has been developed for detection of weak acoustic waves. Improvements in the fringe visibility and sensitivity of the sensor, which uses a low-coherence light source, have been achieved by the introduction of a bandpass optical filter to reduce the spectral width of the interrogating light. Laboratory test results have clearly demonstrated the feasibility of using the fiber sensors for detection of partial discharges. Compared with conventional acoustic sensors, the fiber optic sensor has the advantages of a nonelectrically conducting, high frequency response (up to $200 \mathrm{kHz}$ ), immunity to electromagnetic interference, chemical inertness, small size, and the capability for multiple-point monitoring. Further improvement in performance may be obtainable by use of tunable optical bandpass filters.

The authors acknowledge support for this research from the Electrical Power Research Institute and National Science Funding. The authors also thank Yilu Liu and her students at the Power IT Lab, Virginia Polytechnic Institute, for useful discussions of PD generation and acoustic propagation in transformer oil.

\section{References}

1. Y. Inoue, K. Suganuma, M. Kamba, and M. Kikkawa, "Development of oil-dissolved hydrogen gas detector for diagnosis of transformers," IEEE Trans. Power Deliv. 5, 226-232 (1990).

2. W. A. Pledger and S. C. Pyke, "Gas monitor update: review of progress in the development of a microelectronic in-situ transformer fault gas analyzer," in EPRI Substation Equipment Diagnostics Conference (Electrical Power Research Institute, Palo Alto, Calif., 1994).

3. J. W. Abbott, D. Chu, A. E. Diamond, H. A. ElBadaly, and C. S. Slemon, "Development of an automated transformer oil monitor (ATOM)," in EPRI Substation Equipment Diagnostics Conference (Electrical Power Research Institute, Palo Alto, Calif., 1994).

4. L. E. Lundgaard, "Partial discharge. XIII. Acoustic partial discharge detection-fundamental considerations," IEEE Electr. Insul. Mag. 8(4), 25-31 (1992).

5. L. E. Lundgaard, "Partial discharge. XIV. Acoustic partial discharge detection-practical application," IEEE Electr. Insul. Mag. 8(5), 34-43 (1992).

6. H. Kawada, M. Honda, T. Inoue, and T. Amemiya, "Partial discharge automatic monitor for oil-filled power transformer," IEEE Trans. Power Appar. Syst. PAS-103, 422-428 (1984).

7. E. Howells and E. T. Norton, "Location of partial discharge sites in on-line transformers," IEEE Trans. Power Appar. Syst. PAS-100, 158-161 (1981).

8. P. M. Eleftherion, "Partial discharge. XXI. Acoustic emission-based PD source location in transformers," IEEE Electr. Insul. Mag. 11, 22-26 (1995).

9. B. Culshaw, "Basic concepts of optical fiber sensors," in Optical Fiber Sensors: Principles and Components, J. Dakin and B. Culshaw, eds. (Artech House, Boston, Mass., 1988), Chap. 2.

10. J. A. Bucaro, H. D. Dardy, and E. Carome, "Fiber optic hydrophone," J. Acoust. Soc. Am. 62, 1302-1304 (1977).

11. A. Dandridge and A. D. Kersey, "Overview of Mach-Zehnder 
sensor technology and applications," in Selected Papers on Fiber Optic Sensors, R. Willsch and R. Th. Kersten, eds., Vol. MS 108 of SPIE Milestone Series (Society of Photo-Optical and Instrumentation Engineers, Bellingham, Wash., 1995), pp. 216-234.

12. M. F. Gunther, A. Wang, B. R. Fogg, K. A. Murphy, and R. O. Claus, "Fiber optic impact detection and location system embedded in a composite material," in Fiber Optic Smart Structures and Skins V, R. O. Claus and R. S. Rogowski, eds., Proc. SPIE 1798, 262-269 (1992).

13. J. A. Greene, T. A. Tran, V. Bhatia, M. F. Gunther, A. Wang, K. A. Wang, and R. O. Claus, "Optical fiber sensing technique for impact detection and location in composites and metal specimens," J. Smart Mater. Struct. 4, 93-99 (1995).

14. N. Furstenau, M. Schmidt, H. Horack, W. Goetze, and W. Schmidt, "Extrinsic Fabry-Perot interferometer vibration and acoustic sensor systems for airport ground traffic monitoring," IEE Proc. Optoelectron. 144, 134-144 (1997).

15. J. Teunissen, C. Helmig, R. Merte, and D. Peier, "Fiber optical on-line monitoring for high-voltage transformers," in SPIE International Symposium on Environmental and Industrial Sensing: Fiber Optic Sensor Technology II, M. A. Marcus, B. Culshaw, M. Saad, and J. A. Harrington, eds., Proc. SPIE 4204, 198-205 (2001).

16. Z. Zhao, M. MacAlpine, and M. Süleyman Demokan, "The directionality of an optical fiber high-frequency acoustic sensor for partial discharge detection and location," J. Lightwave Technol. 18, 795-805 (2000).

17. R. O. Claus, M. F. Gunther, A. Wang, and K. A. Murphy, "Extrinsic Fabry-Perot sensor for strain and crack opening displacement measurement from -200 to $900{ }^{\circ}$ C," J. Smart Mater. Struct. 1, 237-242 (1992).

18. K. A. Murphy, M. F. Gunther, A. Wang, and R. O. Claus, "Detection of acoustic emission location using optical fiber sensors," in Smart Structures and Materials 1994: Smart Sensing, Processing, and Instrumentation, J. S. Sirkis, ed., Proc. SPIE 2191, 282-290 (1994).
19. A. Wang, M. S. Miller, A. J. Plante, M. F. Gunther, K. A. Murphy, and R. O. Claus, "Split-spectrum intensity-based optical fiber sensors for measurement of microdisplacement, strain and pressure," Appl. Opt. 35, 2595-2601 (1996).

20. J. Wang, W. Zhao, H. Xiao, and A. Wang, "Self-calibrated interferometric/intensity-based optical fiber sensors," in Sensors and Controls for Advanced Manufacturing, B. O. Nnaji and A. Wang, eds., Proc. SPIE 3201, 20-26 (1998).

21. J. Deng, H. Xiao, W. Huo, M. Luo, R. May, A. Wang, and Y. Liu, "Optical fiber sensor-based detection of partial discharges in power transformers," Opt. Laser Technol. 33, 305-311 (2001).

22. W. Pulliam, P. Russler, R. Mlcak, K. Murphy, and C. Kozikowski, "Micromachined, SiC fiber optic pressure sensors for high temperature aerospace applications," in Industrial Sensing Systems, A. Wang and E. Udd, eds., Proc. SPIE 4202, 21-30 (2000).

23. Y. N. Ning, K. T. V. Grattan, and A. W. Palmer, "Fiber-optic interferometric systems using low-coherence light sources," Sens. Actuators A 30, 181-192 (1992).

24. M. Born and E. Wolf, Principle of Optics (Oxford U. Press, Oxford, 1980), pp. 360-367.

25. M. Schmidt and N. Fürstenau, "Fiber-optic extrinsic FabryPerot interferometer sensors with three-wavelength digital phase demodulation," Opt. Lett. 24, 599-601 (1999).

26. R. A. Wolthuis, G. L. Mitchell, E. Saaski, J. C. Hartl, and M. A. Afromowitz, "Development of medical pressure and temperature sensors employing optical spectrum modulation," IEEE Trans. Biomed. Eng. 38, 974-981 (1991).

27. C. M. Miller, S. C. Mettler, and I. A. White, Optical Fiber Splices and Connectors (Marcel Dekker, New York, 1986), pp. 144-150.

28. M. Di Giovanni, Flat and Corrugated Diaphragm Design Handbook (Marcel Dekker, New York, 1982), Chap. 12.

29. M. Di Giovanni, Flat and Corrugated Diaphragm Design Handbook (Marcel Dekker, New York, 1982), Chap. 17. 